\title{
Discrepancies in ABO and Rh Grouping in Southeast Iran, an Analysis of 3 Years' Experience
}

Esmaei Sannei Moghaddam ${ }^{1}$, Sohail Khosravi ${ }^{1}$ and Akbar Dorgalaleh ${ }^{2^{*}}$

${ }^{1}$ Iranian Blood Transfusion Organization Research Center, Zahedan, Iran

${ }^{2}$ Department of Hematology and Blood Transfusion, Allied Medical School, Tehran University of Medical Sciences, Tehran, Iran

"Corresponding author: Akbar Dorgalaleh, Department of Hematology, Allied Medical School, Tehran University of Medical Sciences, Tehran, Iran, Tel: (+98) 21-32229688; Fax: (+98) 21-883338998; E-mail: dorgalaleha@gmail.com

Received date: Jun 23, 2016, Accepted date: Aug 27, 2016, Publication date: Aug 31, 2016

Copyright: (c) 2016 Moghaddam ES, et al. This is an open-access article distributed under the terms of the Creative Commons Attribution License, which permits unrestricted use, distribution, and reproduction in any medium, provided the original author and source are credited.

\begin{abstract}
Background: $\mathrm{ABO}$ and $\mathrm{Rh}$ blood grouping discrepancies are significant causes of transfusion-related morbidity and mortality. Most of these errors occur when blood grouping standards are unavailable or misinterpreted. This study determined the rate of such errors among hospitals in Zahedan, a city in southeast Iran. We also assessed the prevalence of $\mathrm{ABO}$ and Rh grouping errors in the Iranian Blood Transfusion Organization (IBTO) in Zahedan.
\end{abstract}

Method: During the study, 30,254 blood bags were sent to five of Zahedan's hospitals. Pre-transfusion ABO and $\mathrm{Rh}$ blood grouping was carried out by slide method. Any sample showing a discrepancy between IBTO and hospital laboratory was returned to IBTO for identification of the error by the American Association of Blood Banking (AABB) standards protocol.

Results: We observed 420 discrepancies in the pre-transfusion $A B O$ and Rh blood grouping of 30,254 units, a 1.4 percent error, among Zahedan hospitals. The most common error was misidentification of group A as $O$ (62 cases), while group $B$ was misidentified as $O$ in 41 cases. We discovered critical errors, such as misdiagnosis of $A$ as $B$ and vice versa, which could endanger the patient's life. We also noted 20 misidentifications in routine IBTO laboratory testing, a 0.02 percent error.

Conclusion: The high incidence of pre-transfusion blood grouping errors emphasizes the necessity of always using standard forward and reverse grouping tests in hospitals.

Keywords: ABO; Rh; Blood grouping; Discrepancy

\section{Introduction}

$\mathrm{ABO}$ and $\mathrm{Rh}$ blood group discrepancies account for a considerable number of reported transfusion-associated reactions [1,2]. But the focus now is almost entirely on the risk of transfusion-transmitted infections. Grouping errors can result in significant, even fatal, outcomes. Fortunately, most of these errors do not lead to a clinically adverse outcome. Human errors are a significant contribution to blood grouping discrepancies but some errors are not inevitable [1-3].

The transfusion laboratory is unique among diagnostic laboratories because its life-saving blood products may, at the same time, be lifethreatening agents. Since blood transfusion is a long and complicated process involving many steps and individuals, there is potential for a high rate of error. $\mathrm{ABO}$ and $\mathrm{Rh}$ discrepancy can thus occur $[1,2,4,5]$. These errors have been reported to range from 1 in 3,400 cases to 1 in 517 samples.

Different agents such as miscollection of blood, errors in documentation and registration of patients and donors are responsible $[2,6,7]$. Mindful of these errors, we set up an investigation to determine the rate of $\mathrm{ABO}$ and $\mathrm{Rh}$ grouping discrepancies among hospitals of Zahedan, a city in the southeast of Iran.

\section{Materials and Methods}

This prospective study was carried out on 30,254 donor blood samples in Zahedan's regional blood transfusion center over a period of three years from July 2000 to August 2003. In this period, units of blood sent, on request, to five of Zahedan's hospitals were examined. In hospital blood banks, all samples were rechecked for $\mathrm{ABO}$ and $\mathrm{Rh}$ blood groups by slide method. For this purpose, 5-10 cm segments of tubes were taken from the bags via tube sealers. One drop of corresponding anti sera (human polyclonal anti sera prepared by the IBTO) and one drop of whole blood were mixed in; the reaction was interpreted as agglutination. Units without $\mathrm{ABO}$ and $\mathrm{Rh}$ discrepancy were sent for blood transfusion while any discrepancy resulted in return of the bag to the immunohaematology reference laboratory for further investigation. Initially all samples were rechecked by forward and reverse blood grouping. In the presence of any discrepancy, further investigation, using a protocol as outlined by the AABB manual, was undertaken. Finally obtained results were recorded and the confirmed blood group was reported to each hospital blood bank.

\section{Results}

\section{Incidence of $\mathrm{ABO}$ and $\mathrm{Rh}$ blood group discrepancy}

Out of 30,254 blood donors, 420 cases with $\mathrm{ABO}$ and $\mathrm{Rh}$ blood group discrepancies (1.4\%) were detected. Most were ABO blood 
Citation: Moghaddam ES, Khosravi S, Dorgalaleh A (2016) Discrepancies in ABO and Rh Grouping in Southeast Iran, an Analysis of 3 Years'

Page 2 of 3

group discrepancy (n: 277 cases, 66\%), while Rh comprised 34 percent of total discrepancies (143 cases). Table 1 summarizes the results of $\mathrm{ABO}$ discrepancies.
We also had 4 cases with simultaneous $\mathrm{ABO}$ and $\mathrm{Rh}$ errors, summarized in Table 2.

\begin{tabular}{|l|l|l|l|l|}
\hline Rapid Blood grouping & Forward and reverse & Confirmed diagnosis & Number & Relative percent \\
\hline A & O & O & 19 & 6.9 \\
\hline B & O & O & 22 & 8 \\
\hline AB & O & O & 5 & 2 \\
\hline A & AB & AB & 28 & 10.1 \\
\hline B & AB & AB & 38 & 13.7 \\
\hline O & AB & AB & 4 & 1.4 \\
\hline B & A & A & 18 & 6.5 \\
\hline AB & A & A & 22 & 7.9 \\
\hline O & A & A & 62 & 22.4 \\
\hline A & B & B & 11 & 4 \\
\hline AB & B & B & 7 & 41 \\
\hline O & B & B & 277 & 14.8 \\
\hline & & & 100 \\
\hline
\end{tabular}

Table 1: 277 ABO grouping errors that were observed among five hospitals in Zahedan city.

\begin{tabular}{|l|l|l|l|}
\hline Case & Rapid test & Forward \& Reverse typing & Confirmed test \\
\hline 1 & $\mathrm{AB}+$ & A- & A- \\
\hline 2 & O+ & B- & B- \\
\hline 3 & O- & A+ A+ & B- \\
\hline 4 & $A B+$ & B- & \\
\hline
\end{tabular}

Table 2: Four cases with simultaneous errors of $\mathrm{ABO}$ and Rh blood groups.

The most common $\mathrm{ABO}$ errors were observed in group A blood that was diagnosed as $\mathrm{O}$ (62 cases); B was diagnosed as $\mathrm{O}$ in 41 cases. Other common errors in rapid slide blood grouping concerned the $\mathrm{AB}$ group.

In 38 cases it was diagnosed as $\mathrm{B}$, in 28 cases as $\mathrm{A}$ and in 4 cases as group O. All above errors were due to the use of rapid slide blood grouping. However, we also identified 20 errors made by the IBTO, detected during rapid procedure in hospital pre-transfusion tests.

11 out of 20 were $\mathrm{ABO}$ grouping errors and 9 were $\mathrm{Rh}$ grouping errors. Among IBTO's grouping errors, the most common was the misidentification of $\mathrm{ABO}$ as $\mathrm{B}$. Table 3 summarizes these 11 errors.

\begin{tabular}{|l|l|l|l|l|}
\hline Rapid Blood grouping & Forward and reverse & Confirmed diagnosis & Number & Relative percent \\
\hline AB & B & AB & 4 & 36.3 \\
\hline O & A & O & 3 & 27.3 \\
\hline O & A & O & 3 & 27.3 \\
\hline AB & A & B & 1 & 9.1 \\
\hline & & & 11 & 100 \\
\hline
\end{tabular}

Table 3: Eleven ABO blood grouping errors of IBTO of Zahedan city. 
Citation: Moghaddam ES, Khosravi S, Dorgalaleh A (2016) Discrepancies in ABO and Rh Grouping in Southeast Iran, an Analysis of 3 Years'

Page 3 of 3

Out of 30,254 instances of blood grouping carried out in the IBTO, 20 errors comprised a $0.02 \%$ error rate. Compared with rapid procedure $(1.4 \%)$ this was statistically significant $(<0.001)$.

\section{Discussion and Conclusion}

Discrepancies in blood grouping are the major causes of transfusion reactions. A proper strategy for avoidance of these errors seems critical $[1,3]$. $\mathrm{ABO}$ and $\mathrm{Rh}$ grouping are the most important pre-transfusion tests $[2,5,8]$. Thus this study investigated the prevalence of grouping discrepancies among a number of hospitals and the IBTO, all in Zahedan. All hospitals carried out pre-transfusion $\mathrm{ABO}$ and $\mathrm{Rh}$ grouping by the rapid slide method: we found 1.4 percent of errors in blood grouping by this method. This is a relatively high error rate in a critical situation. If the errors were ignored, they could endanger a patient's life. Some of the misdiagnoses, such as identifying A as B blood group or vice versa, are really critical and dangerous. One important finding was that approximately 39 percent of total errors of the slide method were misdiagnosing blood as group $\mathrm{O}$. This error can occur if, before reading the result, sufficient time is not given for hemagglutination. Another cause for this error is that minor blood groups were not considered as having a weakly positive hemagglutination reaction.

On the other hand, we also detected a 0.02 percent error in IBTO's standard blood grouping. This finding appears minor, but it alarms hospital laboratories as to their absolute confidence in the organization since errors may lead to dangerous results [9-11] such as immediate transfusion reactions.

That some errors creep into routine laboratory work seems inevitable [4,5]. Clerical errors, technical errors, technician observation errors or mistakes in recording the results of standard $\mathrm{ABO}$ and $\mathrm{Rh}$ grouping in regional transfusion organizations can, and do, occur; the possibility of technical or clerical errors cannot be discounted. Thus the necessity for confirmatory grouping tests in hospital before any transfusion. And as rapid blood grouping has a high rate of error and inaccuracy, pre-transfusion grouping by standard procedure can further limit error and the possibility of a transfusion reaction.

\section{Acknowledgment}

Daisy Morant edited the English version of the manuscript.

\section{References}

1. Cangialosi TJ, Riolo ML, Owens S, Dykhouse VJ, Moffitt AH, et al. (2004) The ABO discrepancy index: a measure of case complexity. Am J Orthod Dentofacial Orthop 125: 270-278.

2. Khan MN, Khan TA, Ahmed Z (2013) Discrepancy in ABO Blood Grouping. J Coll Physicians Surg Pak 23: 590-592.

3. Jawitz OK, G Jawitz N, Yuh DD, Bonde P (2013) Impact of ABO compatibility on outcomes after heart transplantation in a national cohort during the past decade. J Thorac Cardiovasc Surg 146: 1239-12946.

4. Milkins C, Berryman J, Cantwell C, Elliott C, Haggas R, et al. (2013) Guidelines for pretransfusion compatibility procedures in blood transfusion laboratories. Transfus Med 23: 3-35.

5. Ansari S, Szallasi A (2011) 'Wrong blood in tube': Solutions for a persistent problem. Vox Sang 100: 298-302.

6. Fastman BR, Kaplan HS (2014) Transfusion-Related Hazards. Patient Safety, Springer. pp: 161-178.

7. Khalid S, Dantes R, Varghese S, Al Hakawati I (2011) Naturally occurring anti $\mathrm{M}$ complicating $\mathrm{ABO}$ grouping. Indian J Pathol Microbiol 54: 170-172.

8. Yazer MH, Hosseini-Maaf B, Olsson ML (2008) Blood grouping discrepancies between $\mathrm{ABO}$ genotype and phenotype caused by $\mathrm{O}$ alleles. Curr Opin Hematol 15: 618-624.

9. Kaur G, Kaur P, Basu S, Kaur R (2014) Blood group discrepancies at a tertiary care centre - analysis and resolution. Int J Lab Hematol 36: 481-487.

10. Chiaroni J, Legrand D, Dettori I, Ferrera V (2004) Analysis of ABO discrepancies occurring in 35 French hospitals. Transfusion 44: 860-864.

11. Sharma T, Garg N, Singh B (2013) ABO blood group discrepancies among blood donors in Regional Blood Transfusion Centre GTB Hospital, Delhi, India. Transfus Apher Sci 50: 75-80. 